\title{
Genome-wide identification, phylogeny, and expression analysis of pectin methylesterases reveal their major role in cotton fiber development
}

Weijie $\mathrm{Li}^{1,2+}$, Haihong Shang ${ }^{1 \dagger}$, Qun Ge${ }^{1}$, Changsong Zou', Juan Cai ${ }^{1}$, Daojie Wang ${ }^{2}$, Senmiao Fan ${ }^{1}$, Zhen Zhang ${ }^{1}$, Xiaoying Deng ${ }^{1}$, Yunna Tan ${ }^{1}$, Weiwu Song ${ }^{1}$, Pengtao Li ${ }^{1}$, Palanga Kibalou Koffi ${ }^{1}$, Muhammad Jamshed ${ }^{1}$, Quanwei Lu', Wankui Gong ${ }^{1}$, Junwen $\mathrm{Li}^{1}$, Yuzhen Shi ${ }^{1}$, Tingting Chen ${ }^{1}$, Juwu Gong ${ }^{1}$, Aiying Liu ${ }^{1}$ and Youlu Yuan ${ }^{1 *}$

\begin{abstract}
Background: Pectin methylesterase (PME, EC 3.1.1.11) is a hydrolytic enzyme that utilizes pectin as substrates, and plays a significant role in regulating pectin reconstruction thereby regulating plant growth. Pectin is one of the important components of the plant cell wall, which forms the main structural material of cotton fiber. In this research, cotton genome information was used to identify PMEs.

Results: We identified 80 (GaPME01-GaPME80) PME genes from diploid G. arboreum (A genome), 78 (GrPME01-GrPME78) PME genes from G. raimondii (D genome), and 135 (GhPME001-GhPME135) PME genes from tetraploid cotton G. hirsutum (AD genome). We further analyzed their gene structure, conserved domain, gene expression, and systematic evolution to lay the foundation for deeper research on the function of PMEs.

Phylogenetic data indicated that members from the same species demonstrated relatively high sequence identities and genetic similarities. Analysis of gene structures showed that most of the PMEs genes had 2-3 exons, with a few having a variable number of exons from 4 to 6 . There are nearly no differences in the gene structure of PMEs among the three (two diploid and one tetraploid) cotton species. Selective pressure analysis showed that the Ka/Ks value for each of the three cotton species PME families was less than one.

Conclusion: Conserved domain analysis showed that PMEs members had a relatively conserved C-terminal pectinesterase domain (PME) while the N-terminus was less conserved. Moreover, some of the family members contained a pectin methylesterase inhibitor (PMEI) domain. The Ka/Ks ratios suggested that the duplicated PMEs underwent purifying selection after the duplication events. This study provided an important basis for further research on the functions of cotton PMEs. Results from qRT-PCR indicated that the expression level of different PMEs at various fiber developmental stages was different. Moreover, some of the PMEs showed fiber predominant expression in secondary wall thickening indicating tissue-specific expression patterns.
\end{abstract}

Keywords: Cotton, Pectin methylesterases (PMEs), Gene family, Gene structure, Phylogeny, Expression patterns

\footnotetext{
* Correspondence: youluyuan@hotmail.com

${ }^{\dagger}$ Equal contributors

${ }^{1}$ State Key Laboratory of Cotton Biology, Key Laboratory of biological and

genetic breeding of cotton, Institute of Cotton Research, Chinese Academy

of Agricultural Sciences, Anyang 455000, Henan, China

Full list of author information is available at the end of the article
} 


\section{Background}

Cotton (Gossypium spp.) is one of the most important natural fiber crops around the world. The improvement of cotton fiber quality is becoming increasingly important and is now a main focal point of cotton research $[1,2]$. Pectin is an important component of cotton fiber and pectin metabolism may influence fiber quality. Previous studies showed that PMEs play an important role in the process of fiber development by influencing the chemical properties of pectin [1]. Process of cotton fiber cell developing was purposely divided into four relative independent but overlapping stages: fiber initiation, elongation, secondary wall biosynthesis and maturation [3]. Fiber initiation and elongation are critical periods in which the number and lengths of fibers, secondary wall thickening (fiber strength), and other fiber quality traits are determined..The secondary wall thickening in cotton fibers starts 15-19 d after flowering and continues to thicken until 40-50d [4]. The increasing thickness of the fiber secondary wall gradually increases the strength of fibers.

A forward subtractive cDNA library constructed and sequenced from upland cotton (G. hirsutum) fibers during the secondary cell wall thickening stage. Computational analysis showed differentially expressed genes that may be involved in cell wall synthesis and modification of biological processes. Among them, several differentially expressed genes which encoded PMEs were identified. Thus, in order to elucidate the relationship between $P M E s$ and fiber development, we analyzed identification, phylogeny expression of PMEs in G. arboreum, G. raimondii and $G$. hirsutum.

PMEs are widely present in plants and some microorganisms that possess a cell wall degradation function. PMEs catalyze the demethylesterification of pectin, which generates carboxyl groups during the release of methanol and hydrogen ions [5]. It plays an important role in cell wall composition modification and degradation if pectin in different development stages of plant, such as fruit maturity [6], pollen development and pollen tube growth [7], cambium cell differentiation, and other plant growth and so on. PMEs have a two-part influence on the cell wall. These produce carboxyl groups and combine with extracellular $\mathrm{Ca}^{2+}$ to form a calcium chain bridge between adjacent pectins, thereby hardening the cell wall and slowing cell diffuse growth [8]. And, the reaction of demethylesterification decreases the extracellular $\mathrm{pH}$ to increase the hydrolytic enzyme activities of enzymes such as poly-galacturonic acid and several pectin enzyme cleavage enzymes [9]. Pectin is subject to substantial degradation, causes cell wall structure relaxation, and enhances the growth of cell tips [10]. The activity of PMEs is regulated by pectin methylesterase inhibitors (PMEIs) [11] whose active site is the conserved PME domain. All members of PME family consist of a catalytically active zone PME domain; some also harbor a PMEI domain. Some proteins containing only one PMEI domain belong to the PMEI family. Therefore, the predicted proteins can be classified into two categories, type I, containing both PME and PMEI domains, and type II, consisting only a PME domain.

The $P M E$ belongs to a multigene family which was first described by Richard [12]. There are 66 PMEs in Arabidopsis [13], 16 in Phytophthora sojae [14], 43 in rice [15], 105 in flax [16], and 81 in G. raimondii [1].

Previous reports suggested that PMEs may play a part in cell wall development of cotton fibers [1]. At present, studies related to $P M E$ genes mainly focused on cloning, and functional analysis of single gene [17]; and few analysis had been carried out at the whole genome level [1]. In 2012, the genome of G. raimondii was completed [18, 19]. The genome map of cultivated cotton G. arboreum was available in 2014 [20]. And next year, the genome map of allotetraploid cultivated cotton (G. hirsutum cv TM-l) was completed [21, 22]. The whole genome sequencing of cotton species provides opportunities for comprehensive analysis and comparison of the PMEs. PMEs, and its homologous genes were analyzed using bioinformatics analysis on the cotton genome sequence. The results showed that sequence similarities and gene structures were highly conserved. In this study, the gene structure, expression, phylogenetic tree, collinearity of homologous genes and other corresponding analysis were examined systematically by employing the methods of bioinformatics. The results of this study will provide novel insights into research of synthesis mechanism of cotton fiber cell wall.

\section{Results}

\section{Identification of cotton PMEs}

From the three cotton genomes (AD, A, and D), we identified 135 full-length putative G. hirsutum PMEs (GhPME001-135), 80 full-length putative G. arboretum PMEs (GaPME01-GaPME80), and 78 full-length putative G. raimondii PMEs (GrPME01-GrPME78) (see Additional file 1: Table S1, and Fig. 1a, c). The family members were named according to their location and sequence on the chromosome.

\section{Gene structure and protein domain of PMEs in different species}

The length of the PMEs between different cotton species was variable mainly due to large differences in the intron length of each gene. The length of the exons in PMEs ranged from 1045 bp to 13398 bp in G. arboreum, $1045 \mathrm{bp}$ to $6730 \mathrm{bp}$ in G. raimondii, and $964 \mathrm{bp}$ to 4695 bp (with a majority between $1500 \mathrm{bp}$ and $2500 \mathrm{bp}$ ) in G. hirsutum (Additional file 1: Table S1). The number of amino acid (AA) residues in the GaPMEs protein 


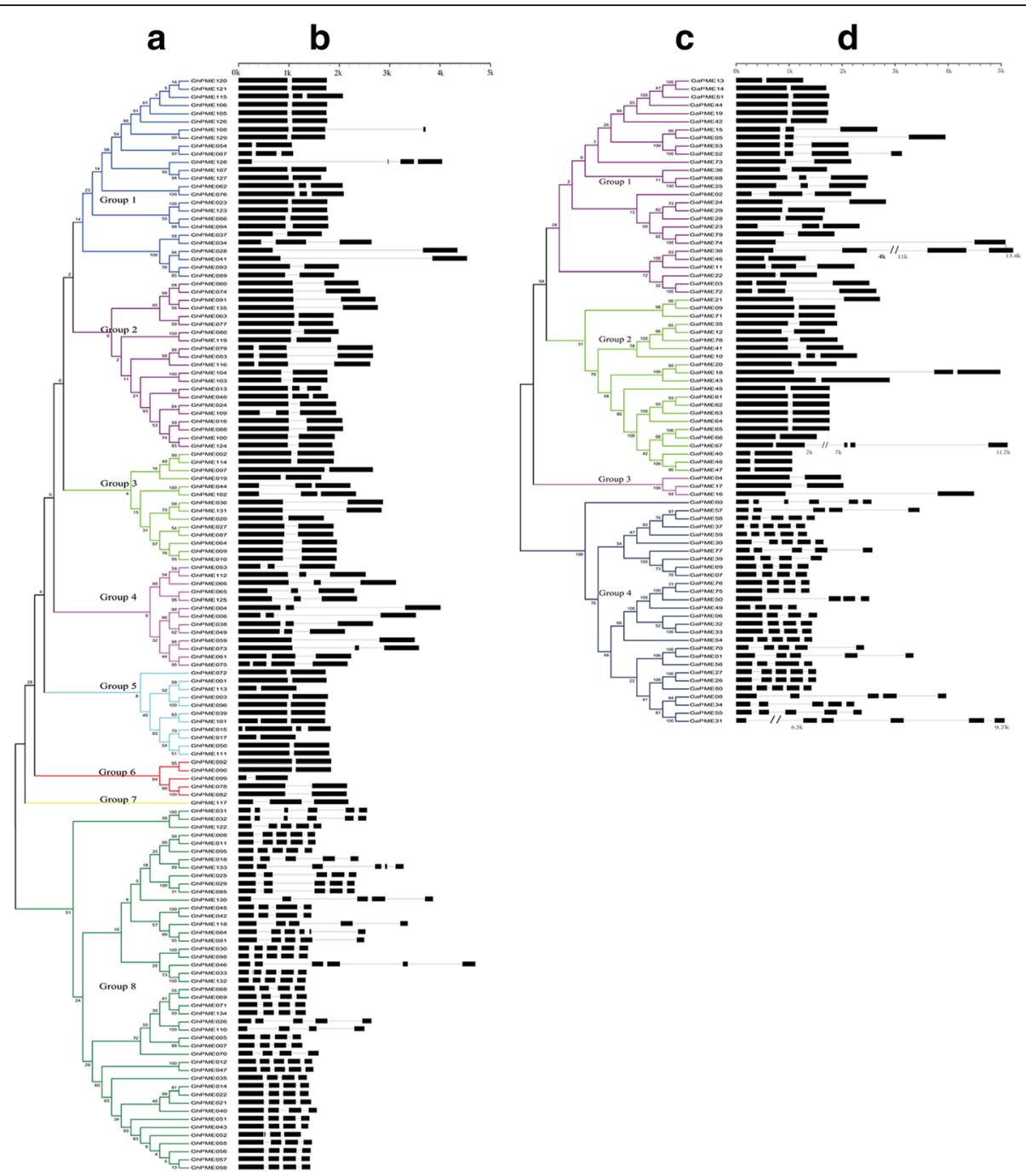

Fig. 1 Phylogenetic relationship and gene structure of the G. arboreum and G. hirsutum PMES. a A phylogenetic tree was constructed using MEGA 5.1 with the neighbor-joining (NJ) method with 1000 bootstrap replicates based on a multiple alignment of 135 amino acid sequences of PMEs from G. hirsutum. The eight major subfamilies are numbered I to VIII. b Exon/intron structure of PMEs from G. hirsutum. Exons and introns are represented by boxes and black lines, respectively. c A phylogenetic tree was constructed with MEGA 5.1 using the neighbor-joining (NJ) method with 1000 bootstrap replicates based on a multiple alignment of 80 amino acid sequences of PMEs from G. arboreum. The four major subfamilies are numbered I to IV. $\mathbf{d}$ Exon/intron structure of PMEs from G. arboreum. Exons and introns are represented by boxes and black lines, respectively

ranged from 301 to 1169,316 to 1644 in GrPMEs, and 260 to 845 in GhPMEs (Additional file 1: Table S1). Asiatic cotton PMEs gene structure analysis results (Fig. 1d) showed that there were differences between different members. The members of the exon number ranged from 2 to 6 , and the gene structure analysis showed that the gene structure of the family members was conserved. The gene structure could be mainly divided into three types. Type I has a typical of two exons and two introns; the differences in the first and second exon were highly conserved, but the length of introns was different. There were 37 such PMEs (46.25\%) distributed in groups one, two, and three. Type II contained three exons, and there were 12 members (15\%) in this group. Among the three exons in this group, the first two exons had significantly different length while the length of the third exon was highly conserved. Type III contained four to six exons with a shorter length than type I or II. These results suggested that the gene structures were similar between of G. hirsutum (Fig. 1b) and G. arboreum (Fig. 1d).

Eighty members of the PMEs family in G. arboreum had evolutionary tree clustering relations, and could be divided into four families (Fig. 1c). The analysis of the conserved sequence of the PMEs family members and domain analysis showed that all of them contain a PME domain. Most of the family members of PMEs contained both PME and PMEI. Only five proteins GaPME13 and GaPME46 in group 1, and GaPME40, GaPME47, and GaPME48 in 
group 2 included only the PME domain. Members of a fourth subfamily contained only a PME domain without a PMEI domain, and there was nearly no difference in $G$. hirsutum (see Additional file 1: Table S1).

\section{Distribution of PMEs family members}

We found 80 PMEs corresponding to the protein-coding genes in the Asiatic cotton database. These 80 genes were distributed on the 13 chromosomes (Additional file 2: Figure S1b), in which the most PMEs (11) were located on chromosome 1 and chromosome 10. Ten PMEs are mapped on chromosome 2, nine on chromosome 9, eight on chromosome 4, three on chromosome 6 , and only one was mapped to chromosome 12 . Each of the chromosomes 3, 5, 7, and 8 had four genes. Only one gene was not detected on the chromosome and was positioned on the scaffold. Eighty genes showed uneven distribution on the chromosomes. Some genes arised by tandem duplication. Seven genes (GaPME61-GaPME67) on chromosome 10 were located on the same block, which we named as cluster I. Five such clusters were located on chromosomes 1, 2, 9, 11, and 13; these clusters covered by $22.5 \%$ of PMEs. We found 135 PMEs in the cotton AD genome (Additional file 2: Figure S1a). Of these, all the 26 chromosomes except At_chr12, fourteen genes $(10.4 \%)$ were located on chromosome Dt_chr9, 10 were mapped on chromosome At_chr9, and some genes appeared via tandem duplication on chromosomes in cotton $\mathrm{AD}$ and $\mathrm{D}$ genomes. We found $78 \mathrm{PMEs}$, of which were distributed to all the chromosomes except chromosomes 4, 12. Chromosomes 9, 8, 6, 7, 2, 1, had $16,10,8,6,2$, and 3 chromosomes respectively. Chromosome 10 and 11 together had five genes; chromosome 5 and 13 had three while chromosomes 3 and 12 had one gene (Additional file 1: Table S1).

Based on the results of collinearity analysis between $G$. raimondii and G. arboreum, 61 homologous gene pairs were distributed in 36 collinearity blocks (Fig. 2a, Additional file 3: Table S2). Among them, one syntonic block contained 19 homologous gene pairs in G. arboreum chromosome 10. We identified 57 homologous gene pairs between $G$. hirsutum (Fig. 2b, Additional file 4: Table S3) and G. arboreum, and 50 homologous gene pairs between G. hirsutum and G. raimondii (Fig. 2b, Additional file 4: Table S3). Some genes were not shown in Fig. 2 because they were not positioned on the chromosome (Additional file 4: Table S3).

\section{Phylogenetic analysis}

Phylogenetic analysis indicated that PMEs of the same species shared the highest similarities and had relatively close genetic relationships. In order to analyze the evolutionary relationships among the predicted GhPMEs, GaPMEs, and GrPMEs based on amino acid sequence, we aligned cotton amino acid sequences with 458 predicted PMEs from eight sequenced plants such as $A$. thaliana, rice, rice, grape, poplar, soybean, cocoa, papaya, and castor bean. Finally, phylogenetic trees were
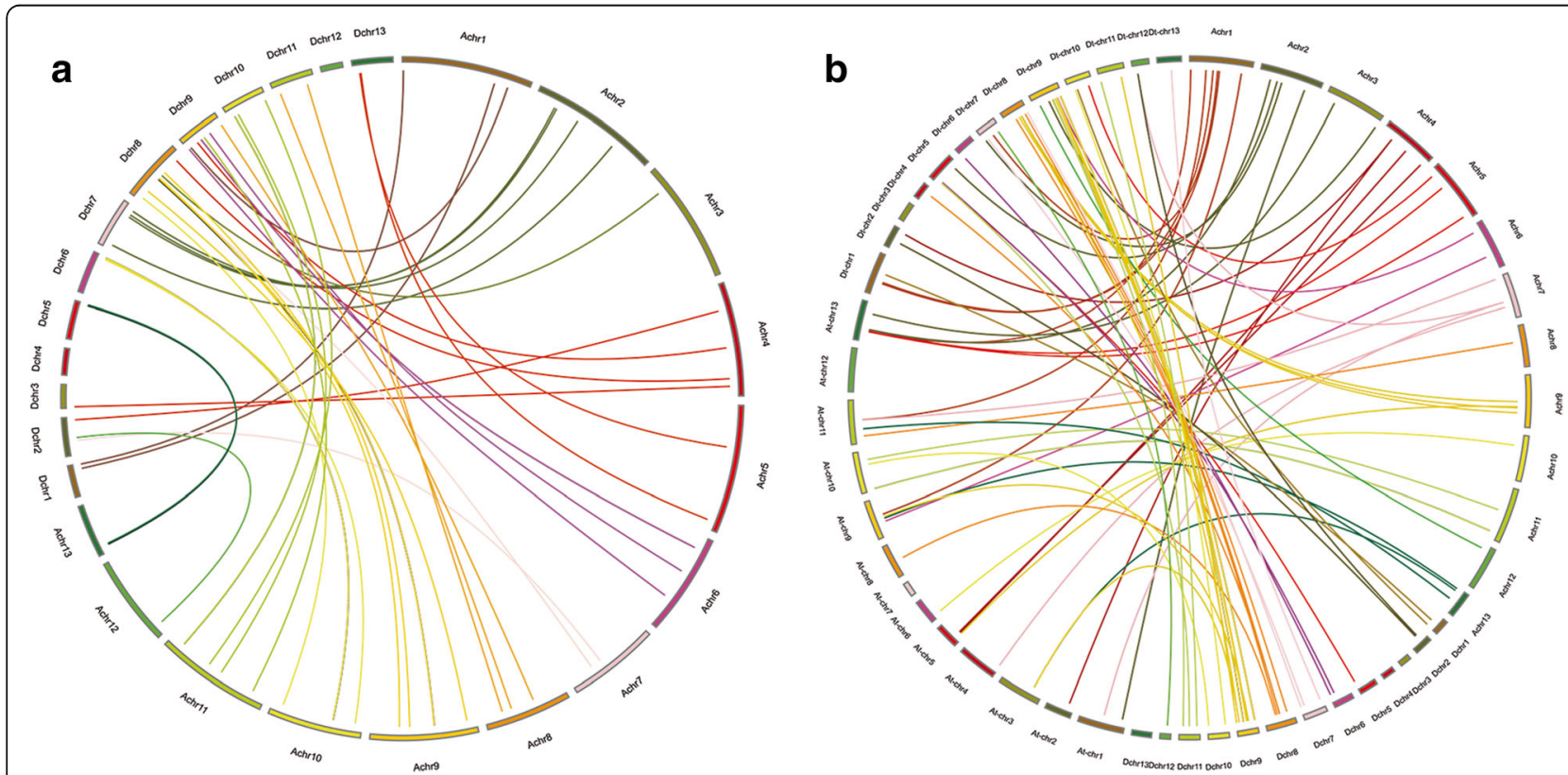

Fig. 2 CIRCOS figure of PME homologous genes pairs of G. raimondii and G. arboreum. a CIRCOS figure of PME homologous genes pairs of G. raimondii and G. arboreum. Lines represent homologous genes that are distributed in syntenic blocks between G. raimondii and G. arboreum chromosomes. b CIRCOS figure of PME homologous genes pairs of G. raimondii and G. hirsutum, G. arboreum and G. hirsutum. Lines represent homologous genes that are distributed in syntenic blocks between G. raimondii, G. arboreum and G. hirsutum chromosomes 
constructed by using MEGA with the neighbor-joining model. We found that the PMEs family could be divided into 10 subfamilies according to cluster analysis (Fig. 3a). The PMEs had a close genetic relationship within the same species and with cocoa. However, these genes were distant to other species on the evolutionary scale. These data indicated that the PMEs might evolve along with different directions for various species. Meanwhile, to examine the evolutionary relationship of PMEs in G. arboreum, G. raimondii, and G. hirsutum, the phylogenetic tree was built with 293 PMEs in which were divided into eight families (Fig. 3b).

The value of the nonsynonymous substitution rate (Ka) to the synonymous substitution rate (Ks) substitutions $(\mathrm{Ka} / \mathrm{Ks})$ can be used as an indicator which could reflect selection pressure of a gene or a gene region during evolution. To infer the influence of selection on the evolution of the three cotton species versus cocoa, we estimated $\mathrm{Ka} / \mathrm{Ks}$ values for all of them (Additional file 5: Table S4). Our results suggested that all of the three cotton species evolved mainly under the influence of stabilizing selection.

\section{Transcriptome analysis}

All of the identified PMEs of G. hirsutum were verified by transcriptome data. Expression of 75.56\% (102 of 135 genes) in G. hirsutum (Fig. 4a) can be detected 0 from 15 day post anthesis (DPA) during fiber development. We detected the expression of $82.5 \%$ (66 of 80) PMEs of G. arboreum (Fig. 4b) and 71.8\% (56 of 78) PMEs of G. raimondii (Fig. 4c) during fiber development (0-15 DPA). We found that 11 PMEs of G. hirsutum (Fig. 4d) and five PMEs of $G$. arboreum were predominantly expressed in fiber development at 15 DPA (belong to the period of secondary wall thickening) (Fig. 4e). However, only three genes in G. raimondii showed higher expression at 15 DPA (Fig. 4f).

To examine the differential expression of homologous gene pairs among the three cotton species, PMEs of G. arboreum predominantly expressed in 15 DPA and its homologous genes were selected for phylogenetic analysis (Fig. 5a). Based on above results, four homologous gene pairs were chose for further studies. The data showed that expression patterns of homologous genes pairs were significantly affected. Moreover, the expression levels of almost all genes in the A genome of cotton were higher than the genes in the $\mathrm{D}$ genome, and lower than the genes in the AD genome of cotton (Fig. 5b), for example, the expression of GrPME23 in fiber development (at 15 days) was 12.28,007, whereas the expression of GaPME17 was 25.40,384. The expression of GhPME037 in fiber development (15 DPA) was 46.41,517. The expression level of GaPME34 was higher than the expression of its orthologous genes in the $\mathrm{AD}$ genome of cotton.

To survey on mechanism of the differences among orthologous gene pairs, we compared their gene structure (Fig. 5c), protein domain conservation (Fig. 5d), and sequence motifs (Fig. 5e). The results showed that orthologous gene pairs have minimal to negligible effect on the structure of the genes. The length of the first exon affected the structure of these genes. GaPME17, GhPME037, and GrPME23, GaPME04 and GhPME089, GaPME34 and GhPME085 were all different in only this one exon. The conserved domain of the protein between the genes did not differ significantly. Protein of orthologous gene pairs varied only on the position of the conserved domain and the length of the non-conserved region (Fig. 5d).

Analysis of putative cis-element motifs of PMEs homologous genes pairs of G. arboreum and G. hirsutum showed significant differences between their promoter regions (Additional file 6: Figure S2). Thus, we speculated that structure variation in promoter region might affect expression levels of homologous gene pairs.

\section{qRT-PCR analysis for PMEs homologous genes pairs}

To verify the alteration of expression patterns of four PMEs homologous gene pairs in G. hirsutum and G. arboreum, qRT-PCR was employed in this study. The results (Fig. 6) showed that the expression of PMEs peaked in Asiatic cotton at 20 DPA and 25 DPA in upland cotton. The average performance of upland cotton was higher than the Asiatic cotton fiber development at 25 DPA suggested that the expression level of Asiatic cotton PMEs was decreased in the late development of cotton fiber. However, in upland cotton, the PMEs expression level continued to increasing. This probably caused the thickening of fibers in the secondary wall, de-esterification of the pectin in the cell wall, reinforcement of the cotton fiber cell wall; thus, increasing the strength and imparting high quality to the upland cotton fiber.

\section{PMEs activity}

There are differences in PMEs activity in different cotton fiber development periods. Of the increasing in Asiatic cotton fiber development and PMEs activity gradually increased from fiber development at 10 DPA to 25 DPA. However, the PMEs activity decreased at 30 DPA. In upland cotton, PMEs activity continued to increasing (Additional file 7: Figure S3). The reason might be that the Asiatic cotton prematurely ended the secondary wall of the fiber growth causing feedback regulation by the cellulose content and accumulation of pectin. 
Li et al. BMC Genomics (2016) 17:1000

Page 6 of 13

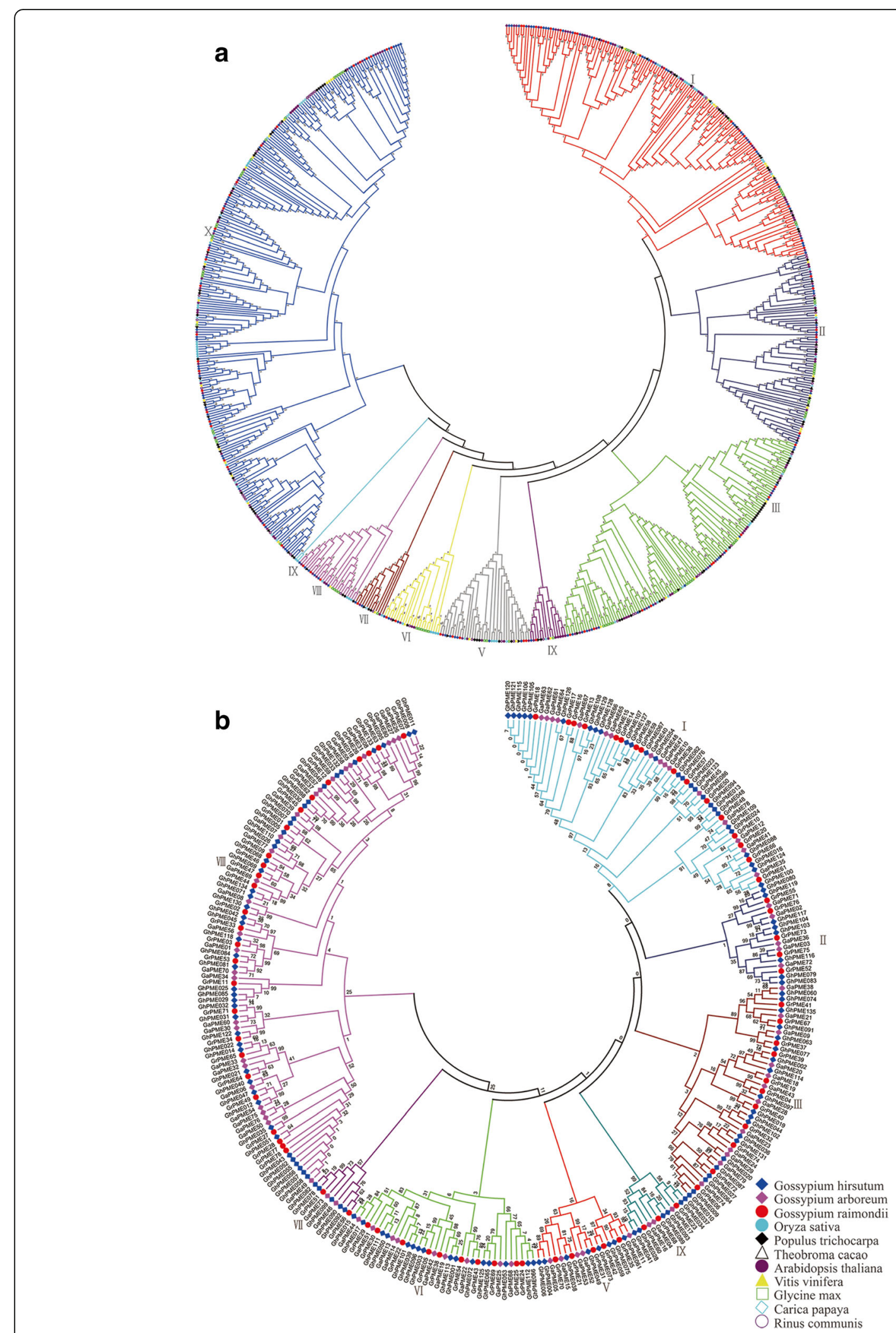

Fig. 3 (See legend on next page.) 
(See figure on previous page.)

Fig. 3 Phylogenetic tree of PMEs. a Phylogenetic tree of PMEs from 11 species. The phylogenetic tree is based on a sequence alignment of the C-terminal PME domains of 751 PMEs protein sequences from 11 genomes, G. hirsutum, G. arboreum, G. raimondii, A. thaliana, O. sativa, V. vinifera, P. trichocarpa, G. max, T. cacao, C. papaya, and R. communis. The PME proteins are grouped into 10 distinct clades (I-X). b Phylogenetic tree of PME domain containing proteins from G. raimondii, G. arboreum, and G. hirsutum. The phylogenetic tree is based on a sequence alignment of the C-terminal PME domains of 293 PME protein sequences from three genomes, G. arboreum, G. raimondii, and G. hirsutum. The tree was generated with MEGA 5.1 using the neighbor-joining method. Bootstrap values from 1000 replicates are indicated at each node. The PME proteins are grouped into 8 distinct clades (I-VIII)

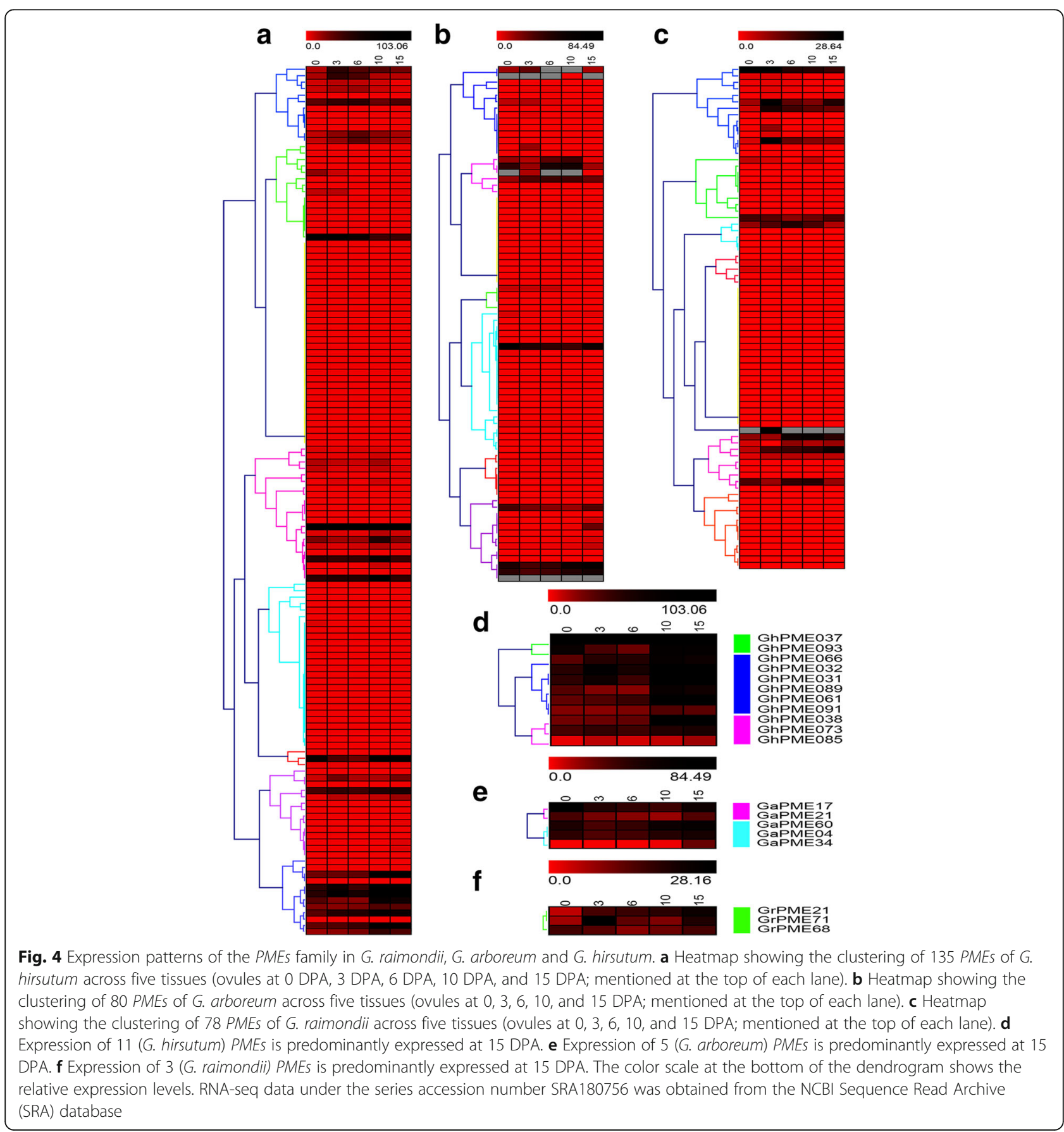




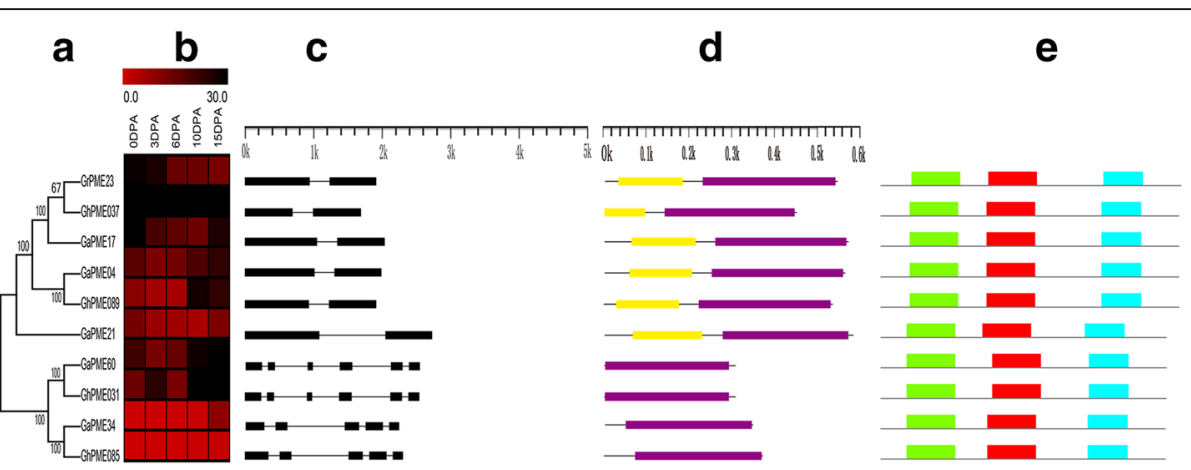

Fig. 5 Analysis of PMEs predominantly expressed in fiber. a The phylogenetic tree was constructed with MEGA 5.1. b Heatmap showing the clustering of PMEs across five tissues (ovules at 0,3,6, 10, and 15 DPA; mentioned at the top of each lane). The color scale at the bottom of the dendrogram shows the relative expression levels. $\mathbf{c}$ Exon/intron structures of PMEs predominantly expressed in fibers. Exons and introns are represented by boxes and black lines, respectively. $\mathbf{d}$ PME domain of the PMEs protein. e Motif of the PME protein

\section{Discussion}

We used bioinformatics analysis to identify 135 GhPME genes from $\mathrm{AD}$ genomes, 80 GaPME genes from A genomes, and 78 GrPME genes from D genomes. Cotton PMEs could be divided into four clades in two diploid species and eight groups in the tetraploid species, and all their last subfamilies were restricted to PME without a PMEI domain. We speculated the common hypothesis that PMEs that both PME and PMEI domains appear relatively late in the evolutionary process [23], similar to the species of that observed in A. thaliana PMEs [24].
Our analysis showed amount of reduction number of genes (from 81 to 78 ) as compared with Liu's study (based on 81 sequences), mainly due to using a more stringent screening criteria. Phylogenetic analysis indicated that these could be divided into four subfamilies (Additional file 8: Figure S4), and the fourth subfamily only contained a PME domain (Additional file 1: Table S1) [1]. Previous studies identified 66, 59, and 89 PMEs coding genes in A. thaliana [24], O. sativa [25], and $P$. trichocarpa [26], respectively. The number of the PMEs varied greatly in different species. Previous studies had
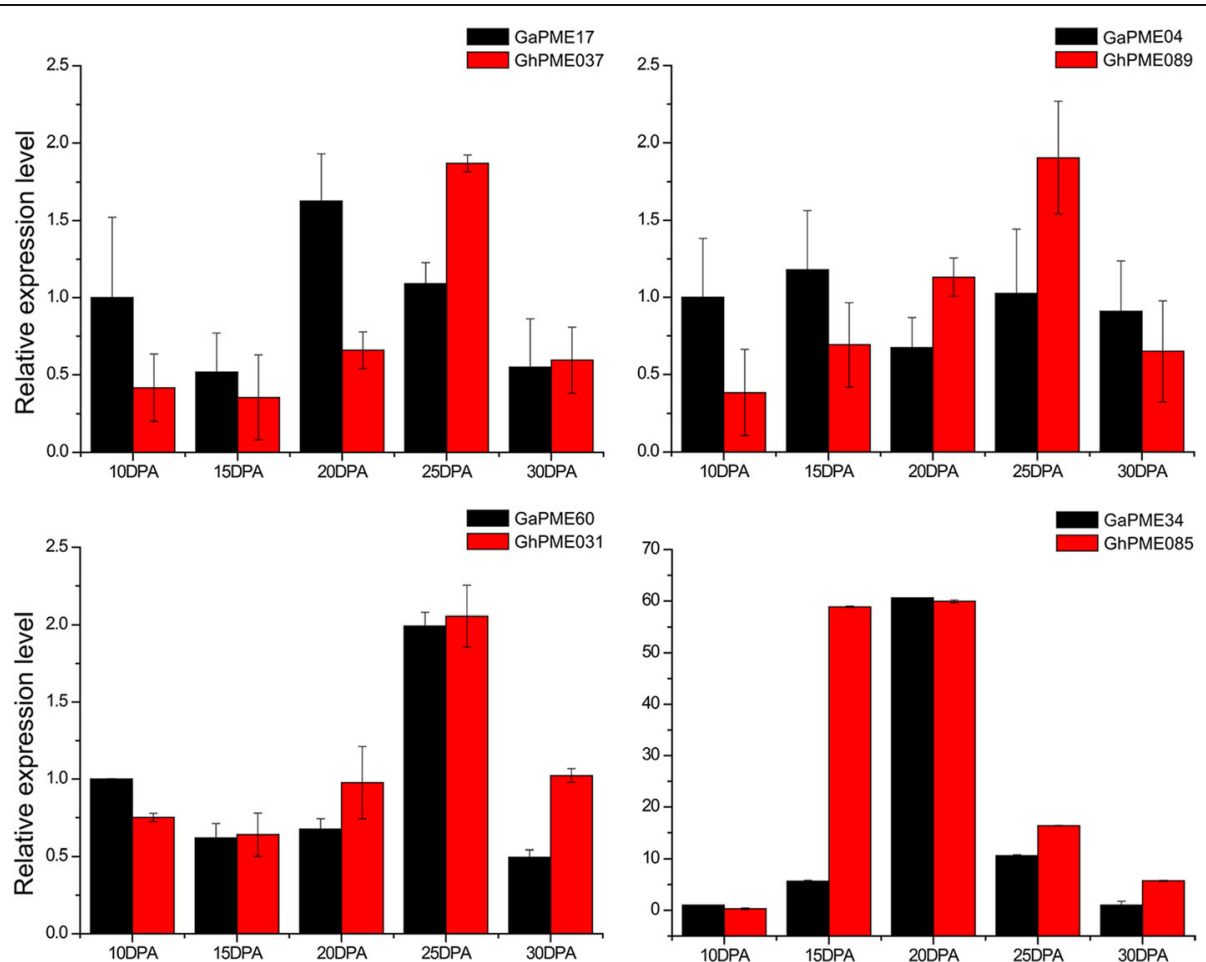

Fig. 6 Expression analysis of 4 selected PMEs homologous genes pairs using RT-qPCR. The relative mRNA abundance of 4 selected PMEs was normalized to the reference gene histone 3 in different tissues. The bars show the standard deviation of three technical replications 
shown that the whole genome duplication (WGD) and tandem repeats were the main reasons of gene family expansion during the process of plant genome evolution $[27,28]$. Plants have a higher rate of gene duplication compared to other eukaryotes [29]. And also, recent studies had shown that genome of diploid cottons had underwent at least two rounds of WGD $[18,19]$. The uneven distribution of genes on the chromosome might be due to gene replication or a partial fragment of gene duplication of the cotton genome that occurred during its long evolutionary history. The entire gene sequence of cotton doubled with every copy event and these extra genes were recombined or go undetected over time [21]. The distribution of genes on the chromosome suggested that 19 of 80 PMEs appeared as tandem repeats in Asiatic cotton with seven members in Cluster I (GaPME61-GaPME67). This was also the main reason for the expansion of this gene family. Arabidopsis $P M E S$ family experienced the $\alpha$ and $\beta$ replication events [25]. Eight genes were formed by tandem duplication; rice PMEs family experienced the events $\sigma$ and $\rho$ copy events, four gene is made copy tandem formation [25].

The evolutionary tree analysis showed that the PMEs sequence within the same species showed high similarity; kinship was also relatively close but was distant from other species. Gene structure analysis showed that most PMEs had 2-3 exons, but a few differed in their number of exons, which ranged from 4 to 6 . The differences in exon numbers might be due to $P M E s$ function and structure as a result of directional evolution. The $\mathrm{N}$ terminus in the evolutionary process was less stringent; some of the family members contain PMEI conserved domains, which might cause changes in the structure and function of PMEs. This conservation helps retain the basic functions of the family, enriches the diversity of genes, and reduces the selection pressure.

Comparative genomics had become a highly interesting area in genomics research especially for the study of extensive genome families. Series of important gene families in crops have been studied by comprehensive analysis, for example, $L E A$ in soybean [29], $L B D$ and $M A P K$ in tomato [27, 28], and MAPKKK in cotton [26]. Previous studies found that PMEs were associated with fiber quality of cotton [1]. Cotton fiber cells are hollow tubular single cells, and their cell wall was the main structure of the mature cotton fiber. Therefore, genes that were directly related to cotton fiber development and regulatory genes, especially the important component of the cell wall-related genes, provided the basis for research and development of cotton fiber and mechanisms affecting its quality. The completion of the cotton genome sequencing enabled the research on PME genes.

PMEs substrate is an important component of the cell wall, which is synthesized in the Golgi complex, and is secreted into the cell wall in the form of methylesters, and quickly de-esterified by PMEs [1]. PMEs plays an important role in regulating plant growth and pectin remodeling [1] Many cell wall-related genes played an important role during cotton cell development [30, 31]. PMEs played a key role in the modification of pectin and formation of the cell wall [16]. Thus, the expression levels of $P M E s$ would likely affect the quality of cotton fiber. Two PMEs, At2g47550, and At4g02330 were cloned from Arabidopsis. At2g47550 was predominantly expressed in pollen grains, and sometimes in vascular tissues. However, the expression of At4g02330 varied in flower and pod throughout their development in Arabidopsis. At4g02330 was mainly expressed in the flower abscission tissue, stigma, microtubule organization, and pollen grains. At2g47550 might be involved in the development of pollen and pollen tube, while At4g02330 might participate in pectin metabolism of cell walls to achieve the regulation of cell separation and petals falling [32]. The effect of PMEs could be reversed during processing (heating) of fruits and vegetables. Fruits and vegetables require $\mathrm{Ca}^{2+}$, which binded to methylester backbone to release carboxyl, and then binds to $\mathrm{Ca}^{2+}$ outside the cell to form a calcium bridge between an adjacent pectin chain, thus hardening the cell wall [33]. Liu et al. verified the five PMEs by studying the PME enzyme activity at different stages of fiber development, pectin content, and demethylation of pectin in Sea Island cotton and Upland cotton [1]. Their results suggested that these genes might be an important factor governing cotton fiber diameter and length [1]. The high expression of G raimondii PME4 and PME5 in fiber development of secondary wall thickening might be related to the cell development [1]. The alignment result showed that all of the cotton PMEs shared high similarity to each other. Moreover, structural similarities suggested that other $P M E$ members in the cotton genome might be associated with the cotton fiber development.

The subfunctionalization of a gene family was prevalent in evolution and gene duplication was the main cause new gene functions [34-36]. Changes in gene expression patterns of the family often occur prior to functional differentiation [34]. This study did not relate to all the features of a PMEs family, but only those involved in cotton fiber development during different periods (0-15DPA). We found that $14(17.5 \%)$ genes were not expressed within a certain time frame suggesting gene redundancy of the copy gene. Gene redundancy raised as a result of interference from the external environment and was important for living systems [37]. Three genes were specifically expressed during cotton fiber elongation indicating their primary role in fiber elongation. PMEs are up-regulated during fiber development suggesting that pectin formation affects fiber diameter 
and length [1], and results in longer and thinner fibers. Pectin could be differentially demethylesterified by PMEs to strengthen or loosen plant cell walls [38]. Five genes were highly expressed in fiber development during secondary wall thickening. Secondary wall thickening was a critical period for the formation of fiber strength. High expression of PMEs during this period was related to the mechanical strength of the fiber.

Expression patterns of orthologous genes pairs were significantly different between two plant species. The expression level of A genome was higher than the expression level of $\mathrm{D}$ genome, the expression level of $G$. hirsutum were higher than the expression of A genome, and only GaPME34 higher than the expression of its orthologous genes. qRT-PCR results (Fig. 6) showed that expression of PMEs genes were difference between diploid and tetraploid cotton. The expression of PMEs in Asiatic cotton and upland cotton peaks (20 DPA in G. arboreum, 25 DPA in G. hirsutum) during fiber development. Of the fiber had a peak, the peak mainly in cotton fiber development 20 DPA. These differences were presumably due to the differences in promoter elements of these genes. The results showed that most PMEs are expressed at high levels in secondary wall thickening of the cotton fiber development, perhaps related to the fiber strength during this period.

PMEs decomposed pectin and played an important role in the expansion process in the plant cell wall. PMEs had different expression patterns in the process of the cotton fiber formation. However, the specific molecular evolutionary mechanisms and posttranscriptional regulation of gene expression pathways and regulatory pathways of PMEs required further investigation.

\section{Conclusions}

This study systematically examined the gene structure, protein domains, physical and chemical properties, gene expression, phylogeny, and collinearity of PMEs. The findings provided here will provide an important basis for further research on the function of cotton PMEs.

\section{Methods}

\section{Materials and processing methods}

The plant material used in this study was G. hirsutum cv 69307 and G. arboreum Shixiya I. The material 69307 were from a RIL population developed with the parents 0-153 and sGK9708 and it showed a positive transgressive segregation in fiber strength. The detail information about the population construction was described in Sun's report [39]. The fiber quality of the parents and the line 69307 was described in Zhang's report [40]. In the day of flowering, flower buds were tagged as zero DPA. The bolls were collected of each sample $(5,10,15,20,25,30 \mathrm{DPA})$ in the morning. The fibers were separated from the ovules, frozen in liquid nitrogen. Before RNA extraction, all the samples were stored at a refrigerator with $-80^{\circ} \mathrm{C}$.

\section{RNA-seq analysis}

CTAB method was used to isolated the subsequent total RNA samples from $3 \mathrm{~g}$ of cotton fiber (G. hirsutum TM-1, G. arboreum Shixiya I and G. raimondii, 0, 3, 6, 10, and 15 DPA) [41]. A Nucleospin ${ }^{\circ}$ RNA clean-up kit (MACHEREYNAGEL, Düren, Germany) was used to purified the total RNA. An Agilent Bio-analyzer (Agilent Technologies, Santa Clara, CA, USA) was used to assess the quality of the RNA sample. After sequencing libraries were prepared following the manufacturer's standard instructions, and all RNA samples were sequenced on an Illumina HiSeq 2500 platform (Illumina, Inc., San Diego, CA, USA). The CLC Genomics Workbench software 4 (http://www.clcbio.com) was used to analyze the transcriptome data with default parameters. $\mathrm{MeV}$ program was used to draw the heat map of the expression data [29].

\section{RNA isolation and qRT-PCR}

The CTAB method was used to extracting the RNA from fiber cell samples [41]. A Nanodrop2000 nucleic acid analyzer was used to test thequality of the RNA sample. A PrimeScript RT reagent kit with a gDNA eraser (TaKaRa, China) was used to performed reverse transcription of samples. The software Premier 5 was used to design primers for the fluorescent quantitative research (Additional file 9: Table S5), and GhHistone 3 (AF024716) was used as a reference gene. The expression levels of the PMEs were measured by using Applied Biosystems $^{\circ} 7500$ Real-Time PCR Systems. Same method was used to analyze expression changes in G. arboreum.

\section{The data related to construct phylogenetic tree}

According to evolutionary analysis of Gossypium, 11 plants sequenced genome were selected for PMEs predicting and further Phylogenetic analysis including $A$. thaliana [42], O. sativa [43], V. vinifera [44], P. trichocarpa [45], G. max [46], T. cacao [47], C. papaya [48], castor bean [49] and three cotton (G. hirsutum [21], G. arboreum [20], and G. raimondii [18]). Annotated protein sequences data sets of 11 plant species with sequenced genome were downloaded from corresponding genome database (see Availability of data and materials). And then, all of the protein sequences were used for identification of PMEs. Predicted PMEs sequences were further phylogenetic tree construction.

\section{Identification of the PME family in cotton}

The software HMMER 3.0 was used to predicted proteins which contained PME (PF01095) (http://pfam.xfam.org) and/or PMEI (PF04043) domains with parameter 
-cut_ga [50]. Only the genes without questionable PFAM annotations (i.e., significant PME and/or PMEI domain but low E-value; low coverage of the domain) were used to do the next analyzes.

\section{Analysis of PMEs family}

The data for the $\mathrm{D}, \mathrm{A}$, and $\mathrm{AD}$ genomes were parsed by a Perl program. The information of PMEs of the calculation of the chromosome locations and structures was selected. We obtained the homologous genes between different cotton species by blast program (E-value $\leq 1 \mathrm{e}$ 10 , Identity $\geq 60 \%$ ). The collinearity of homologous gene pairs was drawn using CIRCOS package (http://circos.ca). Mapping of $P M E$ genes was performed using Map Chart [51]. Phylogenetic trees were constructed by employing MEGA software with neighbor joining model, and bootstrap values (1000 replicates) are indicated at each node [52]. Exons and introns were displayed by using The Gene Structure Display Server (GSDS, http:// gsds.cbi.pku.edu.cn) [53]. Conserved domains prediction were performed using the SMART Package (http://smart.embl-heidelberg.de) program [54]. Motif analysis was conducted using online tools (maximum number of motifs, three; minimum motif width, six; and maximum motif width, 50) (http://meme.nbcr.net/meme) [55]. The cis-acting elements prediction was performed using an online tool PlantCARE (http://bioinformatics.psb.ugent.be/webtools/plantcare/html) [56].

\section{PMEs activity assay}

Based on the Hagerman with some modifications [57], total PMEs enzyme activity was measured. Briefly, $1 \mathrm{~g}$ of a fiber sample was taken in a prechilled mortar and $5.0 \mathrm{~mL}$ $8.8 \%(\mathrm{w} / \mathrm{v})$ pre-cooled $\mathrm{NaCl}$ was added. The samples were centrifuged for $10 \mathrm{~min}$; the supernatant was collected, and adjusted to $\mathrm{pH}=7.5$ with $0.1 \mathrm{~mol} / \mathrm{L} \mathrm{NaOH}$ to obtain a crude enzyme solution. Four milliliters of $0.5 \%(\mathrm{w} / \mathrm{v}) \mathrm{pec}-$ tin solution and $0.3 \mathrm{~mL} 0.01 \%(\mathrm{w} / \mathrm{v})$ bromophenol blue were added to a test-tube, followed by adding $300 \mathrm{uL}$ of an enzyme solution. After $2 \mathrm{~min}$, the absorbance value, and the $\Delta A 620 / \mathrm{min} \cdot g$ expressed enzyme activity, of each sample was analyzed in triplicate [57].

\section{Additional files}

Additional file 1: Table S1. PMEs genes identified from the three cotton species. (XLSX $36 \mathrm{~kb}$ )

Additional file 2: Figure S1. Chromosomal location of PMEs. a. Chromosomal location of 135 GhPME genes. A total of 104 genes are located on normal chromosomes, whereas the other 31 are located on scaffolds. b. Chromosomal location of 80 GaPME genes. A total of 79 genes are located on normal chromosomes, whereas the other one is located on scaffolds. (TIF $1445 \mathrm{~kb}$ )

Additional file 3: Table S2. Orthologous PMEs gene pairs of $G$. arboreum and G. raimondii. (XLSX $14 \mathrm{~kb}$ )
Additional file 4: Table S3. Orthologous PMEs gene pairs of the three cotton species. (XLSX $23 \mathrm{~kb}$ )

Additional file 5: Table S4. Selective pressure between T. cacao L. and the three cotton species paralogous PMEs gene pairs. (XLSX $23 \mathrm{~kb}$ )

Additional file 6: Figure S2. Analysis of putative cis-element motifs of PME homologous genes pairs of G. arboreum and G. hirsutum promoter. cis-element motifs are represented by boxes. (TIF $612 \mathrm{~kb}$ )

Additional file 7: Figure S3. Cotton fiber proteins were isolated and used for PME activity assay. Error bars represent the SE of three biological replicates. (TIF $173 \mathrm{~kb}$ )

Additional file 8: Figure S4. A phylogenetic tree was constructed with MEGA 5.1 using the neighbor-joining (NJ) method with 1000 bootstrap replicates based on a multiple alignment of 78 amino acid sequences of PMEs from G. raimondii. The four major subfamilies are numbered I to IV. (TIF 1228 kb)

Additional file 9: Table S5. Primer pairs used in quantitative real-time PCR analysis. (DOCX $12 \mathrm{~kb})$

\section{Abbreviations}

AA: Amino acid; DPA: Day post anthesis; Ga: Gossypium arboreum; Gb: Gossypium barbadense; Gh: Gossypium hirsutum; Gr: Gossypium raimondii; Ka: Nonsynonymous substitution rate; Ks: Synonymous substitution rate; LEA: Late embryogenesis abundant; MAPK: Mitogen aetivated protein kinase; MAPKKK: Mitogen activated protein kinase kinase kinase; PME: Pectin methylesterase; PMEl: Pectin methylesterase inhibitor; qRT-PCR: Quantitative real-time polymerase chain reaction; WGD: Whole genome duplication

\section{Acknowledgments}

Thank you to Dr. Daojie Wang at the Institute of Henan University for his insightful comments while preparing the manuscript.

\section{Funding}

This work was supported by National Natural Science Foundation of China (31471538), and the National High Technology Research and Development Program of China (2013AA102601).

\section{Availability of data and materials}

G. hirsutum, G. arboreum, and G. raimondii (http://cgp.genomics.org.cn), A. thaliana (http://www.arabidopsis.org), O. sativa (http://rapdb.dna.affrc.go.jp), V. vinifera (http://www.genoscope.cns.fr/spip/Vitis-vinifera-e.html), P. s trichocarpa (http://www.phytozome.net/poplar), G. max (http:// www.phytozome.net/soybean), T. cacao L. (http://cocoagendb.cirad.fr), C. papaya (http://asgpb.mhpcc.hawaii.edu), and castor bean (http:// castorbean.jcvi.org). The phylogenetic data was uploaded to Treebase under Accession URL: http://purl.org/phylo/treebase/phylows/study/TB2:S20141.

\section{Authors' contributions}

YLY, HHS, and QG designed the experiments, WJL conducted gene expression analyses and drafted the manuscript. HHS, and CSZ provided the RNA-seq data. JC and SMF participated in the sequence alignment and PMEs activity test. ZZ, XYD, YNT, WWS, PTL, QWL, PKK, and MJ performed phylogenetic analyses of PMEs. DJW, WKG, YZS, TTC, and JWL helped to draft the manuscript. JWG and AYL prepared the cotton. All authors read and approved the final manuscript.

\section{Competing interests}

The authors declare that they have no competing interests.

\section{Consent for publication}

Not applicable.

\section{Ethics approval and consent to participate}

Not applicable.

\section{Author details}

${ }^{1}$ State Key Laboratory of Cotton Biology, Key Laboratory of biological and genetic breeding of cotton, Institute of Cotton Research, Chinese Academy of Agricultural Sciences, Anyang 455000, Henan, China. ${ }^{2}$ State Key Laboratory 
of Cotton Biology, Key Laboratory of Plant Stress Biology, College of Life Science, Henan University, Kaifeng 475004, China.

\section{Received: 27 February 2016 Accepted: 2 December 2016 Published online: 07 December 2016}

\section{References}

1. Liu Q, Talbot M, Llewellyn DJ. Pectin methylesterase and pectin remodelling differ in the fibre walls of two gossypium species with very different fibre properties. PLoS One. 2013;8(6):e65131.

2. Jamshed M, Jia F, Gong J, Palanga KK, Shi Y, Li J, Shang H, Liu A, Chen T, Zhang Z. Identification of stable quantitative trait loci (QTLs) for fiber quality traits across multiple environments in Gossypium hirsutum recombinant inbred line population. BMC Genomics. 2016;17(1):1.

3. Basra AS, Malik C. Development of the cotton fiber. Int Rev Cytol. 1984:89(1):65-113.

4. Wilkins $T A$, Arpat $A B$. The cotton fiber transcriptome. Physiol Plant. 2005;124(3):295-300

5. Jolie RP, Duvetter T, Van Loey AM, Hendrickx ME. Pectin methylesterase and its proteinaceous inhibitor: a review. Carbohydr Res. 2010:345(18):2583-95.

6. Kagan-Zur V, Tieman DM, Marlow SJ, Handa AK. Differential regulation of polygalacturonase and pectin methylesterase gene expression during and after heat stress in ripening tomato (Lycopersicon esculentum Mill.) fruits. Plant Mol Biol. 1995:29(6):1101-10.

7. Bosch M, Cheung AY, Hepler PK. Pectin methylesterase, a regulator of pollen tube growth. Plant Physiol. 2005;138(3):1334-46.

8. Qin Y-M, Zhu Y-X. How cotton fibers elongate: a tale of linear cell-growth mode. Curr Opin Plant Biol. 2011;14(1):106-11.

9. Wen F, Zhu Y, Hawes MC. Effect of pectin methylesterase gene expression on pea root development. Plant Cell. 1999;11(6):1129-40.

10. Catoire LC L, Pierron M, Morvan C, du Penhoat CH, Goldberg R. Investigation of the action patterns of pectinmethylesterase isoforms through kinetic analyses and NMR spectroscopy - Implications in cell wall expansion. J Biol Chem. 1998;50:33150-6.

11. BALESTRIERI C, CASTALDO D, GIOVANE A, QUAGLIUOLO L, SERVILLO L. A glycoprotein inhibitor of pectin methylesterase in kiwi fruit (Actinidia chinensis). Eur J Biochem. 1990;193(1):183-7.

12. Richard L, Qin L-X, Gadal P, Goldberg R. Molecular cloning and characterisation of a putative pectin methylesterase CDNA in Arabidopsis thaliana (L.). FEBS Lett. 1994;355(2):135-9.

13. Louvet R, Cavel E, Gutierrez L, Guénin S, Roger D, Gillet F, Guerineau F, Pelloux J. Comprehensive expression profiling of the pectin methylesterase gene family during silique development in Arabidopsis thaliana. Planta. 2006;224(4):782-91.

14. Horowitz BB, Ospina-Giraldo MD. The pectin methylesterase gene complement of phytophthora sojae: structural and functional analyses, and the evolutionary relationships with its oomycete homologs. PLoS One. 2015;10(11):e0142096.

15. Jeong HY, Nguyen HP, Lee C. Genome-wide identification and expression analysis of rice pectin methylesterases: Implication of functional roles of pectin modification in rice physiology. J Plant Physiol. 2015;183:23-9.

16. Pinzón-Latorre D, Deyholos MK. Characterization and transcript profiling of the pectin methylesterase (PME) and pectin methylesterase inhibitor (PMEI) gene families in flax (Linum usitatissimum). BMC Genomics. 2013;14(1):742.

17. Tingting $C$, Xukai L, Ruyi W, Liangcai $P$, Tao X. Cloning and expression analysis of GhPME 1 and GhPME 2 in cotton. J China Agric Univ. 2012;17(005):7-14.

18. Wang K, Wang Z, Li F, Ye W, Wang J, Song G, Yue Z, Cong L, Shang H, Zhu $\mathrm{S}$, et al. The draft genome of a diploid cotton Gossypium raimondii. Nat Genet. 2012;44(10):1098-103.

19. Paterson AH, Wendel JF, Gundlach H, Guo H, Jenkins J, Jin D, Llewellyn D, Showmaker KC, Shu S, Udall J. Repeated polyploidization of Gossypium genomes and the evolution of spinnable cotton fibres. Nature. 2012;492(7429):423-7.

20. Li F, Fan G, Wang K, Sun F, Yuan Y, Song G, Li Q, Ma Z, Lu C, Zou C. Genome sequence of the cultivated cotton Gossypium arboreum. Nat Genet. 2014;46(6):567-72.

21. Li F, Fan G, Lu C, Xiao G, Zou C, Kohel RJ, Ma Z, Shang H, Ma X, Wu J, et al. Genome sequence of cultivated Upland cotton (Gossypium hirsutum TM-1) provides insights into genome evolution. Nat Biotechnol. 2015;33(5):524-30.
22. Zhang T, Hu Y, Jiang W, Fang L, Guan X, Chen J, Zhang J, Saski CA, Scheffler BE, Stelly DM, et al. Sequencing of allotetraploid cotton (Gossypium hirsutum L. acc. TM-1) provides a resource for fiber improvement. Nat Biotechnol. 2015;33(5):531-7.

23. Micheli F. Pectin methylesterases: cell wall enzymes with important roles in plant physiology. Trends Plant Sci. 2001:6(9):414-9.

24. Hongo S, Sato K, Yokoyama R, Nishitani K. Demethylesterification of the Primary Wall by PECTIN METHYLESTERASE35 Provides Mechanical Support to the Arabidopsis Stem. Plant cell. 2012;24:2624-34.

25. Wang M, Yuan D, Gao W, Li Y, Tan J, Zhang X. A comparative genome analysis of PME and PMEl families reveals the evolution of pectin metabolism in plant cell walls. PLoS One. 2013;8(8):1-12.

26. Markovič O, Janeček Š. Pectin methylesterases: sequence-structural features and phylogenetic relationships. Carbohydr Res. 2004;339(13):2281-95.

27. Chothia C, Gough J, Vogel C, Teichmann SA. Evolution of the protein repertoire. Science. 2003;300(5626):1701-3.

28. Ohno $\mathrm{S}$, Wolf $\mathrm{U}$, Atkin NB. Evolution from fish to mammals by gene duplication. Hereditas. 1968;59(1):169-87.

29. Wei K, Wang Y, Xie D. Identification and expression profile analysis of the protein kinase gene superfamily in maize development. Mol Breed. 2014:33(1):155-72.

30. Salnikov W, Grimson MJ, Seagull RW, Haigler CH. Localization of sucrose synthase and callose in freeze-substituted secondary-wall-stage cotton fibers. Protoplasma. 2003;221(3-4):175-84.

31. Ruan Y-L, Llewellyn DJ, Furbank RT. Suppression of sucrose synthase gene expression represses cotton fiber cell initiation, elongation, and seed development. Plant Cell. 2003;15(4):952-64.

32. Zhang L, Xue J, Yu H, Li Q, Li R. The expression and function study of pectin methylesterase genes which regulate and control the petal falling in Arabidopsis. Plant Physiol J. 2012:48(4):350-8.

33. Yeom HW, Streaker CB, Zhang QH, Min DB. Effects of pulsed electric fields on the quality of orange juice and comparison with heat pasteurization. J Agric Food Chem. 2000;48(10):4597-605.

34. Ohno S. Evolution by gene duplication. Department of Biology City of Hope Medical Center Duarte. New York: Springer Science \& Business Media; 2013.

35. Hughes AL. The evolution of functionally novel proteins after gene duplication. Proc R Soc Lond B Biol Sci. 1994;256(1346):119-24.

36. Moore RC, Purugganan MD. The evolutionary dynamics of plant duplicate genes. Curr Opin Plant Biol. 2005;8(2):122-8.

37. Zheng X, Zhu J, Kapoor A, Zhu JK. Role of Arabidopsis AGO6 in siRNA accumulation, DNA methylation and transcriptional gene silencing. EMBO J. 2007:26(6):1691-701.

38. Levesque-Tremblay G, Müller K, Mansfield SD, Haughn GW. HIGHLY METHYL ESTERIFIED SEEDS is a pectin methyl esterase involved in embryo development. Plant Physiol. 2015;167(3):725-37.

39. Sun F-D, Zhang J-H, Wang S-F, Gong W-K, Shi Y-Z, Liu A-Y, Li J-W, Gong J-W, Shang H-H, Yuan Y-L. QTL mapping for fiber quality traits across multiple generations and environments in upland cotton. Mol Breed. 2011;30(1):569-82.

40. Zhang Z, Li J, Muhammad J, Cai J, Jia F, Shi Y, Gong J, Shang H, Liu A, Chen $\mathrm{T}$, et al. High resolution consensus mapping of quantitative trait loci for fiber strength, length and micronaire on chromosome 25 of the upland cotton (Gossypium hirsutum L.). PLoS One. 2015;10(8):e0135430.

41. Ji SJ, Lu YC, Feng JX, Wei G, Li J, Shi YH, Fu Q, Liu D, Luo JC, Zhu YX. Isolation and analyses of genes preferentially expressed during early cotton fiber development by subtractive PCR and CDNA array. Nucleic Acids Res. 2003;31(10):2534-43.

42. Cao J, Schneeberger K, Ossowski S, Gunther T, Bender S, Fitz J, Koenig D, Lanz C, Stegle O, Lippert C, et al. Whole-genome sequencing of multiple Arabidopsis thaliana populations. Nat Genet. 2011;43(10):956-63.

43. Goff SA, Ricke D, Lan T-H, Presting G, Wang R, Dunn M, Glazebrook J, Sessions A, Oeller P, Varma $\mathrm{H}$, et al. A draft sequence of the rice genome (Oryza sativa L. ssp. Japonica). Science. 2002;296:79-99.

44. Jaillon O, Aury JM, Noel B, Policriti A, Clepet C, Casagrande A, Choisne N, Aubourg S, Vitulo N, Jubin C, et al. The grapevine genome sequence suggests ancestral hexaploidization in major angiosperm phyla. Nature. 2007:449(7161):463-7.

45. Tuskan GA, Difazio S, Jansson S, Bohlmann J, Grigoriev, Hellsten, Putnam N, Ralph S, Rombauts, Salamov A, et al. The genome of black cottonwood, Populus trichocarpa (Torr. \& Gray). Science. 2006;313:1596-604.

46. Schmutz J, Cannon SB, Schlueter J, Ma J, Mitros T, Nelson W, Hyten DL, Song Q, Thelen JJ, Cheng J, et al. Genome sequence of the palaeopolyploid soybean. Nature. 2010;463(7278):178-83. 
47. Argout X, Salse J, Aury JM, Guiltinan MJ, Droc G, Gouzy J, Allegre M, Chaparro C, Legavre T, Maximova SN, et al. The genome of Theobroma cacao. Nat Genet. 2011;43(2):101-8.

48. Ming R, Hou S, Feng Y, Yu Q, Dionne-Laporte A, Saw JH, Senin P, Wang W, Ly BV, Lewis KL, et al. The draft genome of the transgenic tropical fruit tree papaya (Carica papaya Linnaeus). Nature. 2008:452(7190):991-6.

49. Chan AP, Crabtree J, Zhao Q, Lorenzi H, Orvis J, Puiu D, Melake-Berhan A, Jones KM, Redman J, Chen G, et al. Draft genome sequence of the oilseed species Ricinus communis. Nat Biotechnol. 2010;28(9):951-6.

50. Finn RD, Clements J, Eddy SR. HMMER web server: interactive sequence similarity searching. Nucleic Acids Res. 2011;39(Web Server issue):W29-37.

51. Voorrips RE. MapChart: software for the graphical presentation of linkage maps and QTLs. J Hered. 2002;93(1):77-8.

52. Tamura K, Peterson D, Peterson N, Stecher G, Nei M, Kumar S. MEGA5: molecular evolutionary genetics analysis using maximum likelihood, evolutionary distance, and maximum parsimony methods. Mol Biol Evol. 2011;28(10):2731-9.

53. Hu B, Jin J, Guo A-Y, Zhang H, Luo J, Gao G. GSDS 2.0: an upgraded gene feature visualization server. Bioinformatics. 2015;31(8):1296-7.

54. Letunic I, Doerks T, Bork P. SMART: recent updates, new developments and status in 2015. Nucleic Acids Res. 2015;43(D1):D257-60.

55. Bailey TL, Williams N, Misleh C, Li WW. MEME: discovering and analyzing DNA and protein sequence motifs. Nucleic Acids Res. 2006;34(Web Server issue):W369-73.

56. Lescot M, Dehais P, Thijs G, Marchal K, Moreau Y, Van de Peer Y, Rouze P, Rombauts $\mathrm{S}$. PlantCARE, a database of plant cis-acting regulatory elements and a portal to tools for in silico analysis of promoter sequences. Nucleic Acids Res. 2002;30(1):325-7.

57. Hagerman AEA, Paul J. Continuous spectrophotometric assay for plant pectin methyl esterase. J Agric Food Chem. 1986;34:440-4.

\section{Submit your next manuscript to BioMed Central and we will help you at every step:}

- We accept pre-submission inquiries

- Our selector tool helps you to find the most relevant journal

- We provide round the clock customer support

- Convenient online submission

- Thorough peer review

- Inclusion in PubMed and all major indexing services

- Maximum visibility for your research

Submit your manuscript at www.biomedcentral.com/submit

) Biomed Central 